Ostracoda is one of the most diverse groups of crustaceans living in all aquatic ecosystems: marine, brackish, and freshwater (including subterranean ecosystems). They are cosmopolitan in distribution OPEN ACCESS and play a vital role in the food chain and energy flow in the aquatic ecosystem. Ostracoda is considered as a separate class under Crustacea and it has been divided into the subclasses Myodocopa (only marine representatives) and Podocopa (Martin \& Davis 2001; Horne et al. 2002). The subclass Podocopa has the following three orders: Platycopida, which includes marine and a very few brackish water forms; Podocopida which is present in both freshwater and the marine environment; and Palaeocopida known only from fossils records (Karanovic 2012). Globally, there are about 2103 species of non-marine Ostracoda and they are classified into 209 genera, 15 families and three superfamilies: Darwinuloidae, Cytheroidea, and Cypridoidea (Martens \& Savatenalinton 2011; Karanovic 2012). Among the different zoogeographic regions, the oriental region has 200 species, of which $83 \%$ are endemic and the Australian region alone accounts for 176 species (Martens et al. 2008). Freshwater habitat exhibits a high Ostracoda diversity inspite of a rapid decline in freshwater biodiversity (Dudgeon et al. 2006, Martens \& Savatenalinton 2011).

The taxonomic studies on Indian Ostracods were initiated by Baird (1859). Later, Victor \& Fernando (1979) listed about 56 freshwater Ostracoda species

\section{FreshwATER Ostracoda (CRUstacea) OF INDIA - A CHECKLIST}

\section{Karuthapandi ${ }^{1}$, D.V. Rao ${ }^{2} \&$ B. Xavier Innocent ${ }^{3}$}

${ }^{1,2}$ Freshwater Biological Regional Center, Zoological Survey of India, Plot No.-366/1, Attapur(V), Near O/o DFO Flying Squad Hyderguda, P.O. Ring Road, Hyderabad, Telangana 500048, India ${ }^{3}$ Department of Zoology, St. Xavier's College (Autonomous), Palayamkottai, Tamil Nadu 627002, India ${ }^{1}$ kpandi83@gmail.com (corresponding author), 2drraodv@gmail.com, ${ }^{3}$ xiyuvaraj1010@gmail.com

from the Indian subcontinent. Further, Venkataraman \& Krishnamoorthy (1998) estimated that there were 120 species of Ostracoda from freshwater and marine habitats, which included 24 genera and four families, but there was no comprehensive list. Therefore, based on the review of published data in Baird (1859), Gurney (1907), Klie (1927), Arora (1931), Brehm (1950), Hartmann (1964), Deb (1972, 1973a,b,c, 1983), Victor \& Michael (1975), Victor \& Fernando $(1979,1981)$, Battish (1978, 1981, 1998), Harshey \& Victor (1983), Harshey \& Shrivastava (1983), Gupta (1984, 1988 \& 1991), Harshey \& Srinivasan (1984), Harshey et al. (1987), Harshey \& Patil (1988), Harshey (1996, 2008), George (1993), Jayasree et al. (1994) Venkataraman (1999), Patil (2002), George \& Martens (2002, 2003, 2004), Patil \& Talmale (2005a,b, 2012), Karanovic \& Reddy (2008) and Harshey \& Thilak (2011), we compile a checklist of Indian Ostracoda and discuss their status.

In the present review of Indian freshwater ostracods, we list 152 valid species belonging to 39 genera, five families and two super families (Tables 1,2). Further,

DOI: http://dx.doi.org/10.11609/JoTT.o3682.6576-81 | ZooBank: urn:Isid:zoobank.org:pub:1BFB200A-B63E-4B10-938F-23345E78C920

Manuscript details: Ms \# 03682 | Received 26 June 2013 | Final received 12 September 2014 | Finally accepted 28 October 2014

Citation: Karuthapandi, M., D.V. Rao \& B.X. Innocent (2014). Freshwater Ostracoda (Crustacea) of India - a checklist. Journal of Threatened Taxa 6(12): 6576-6581; http://dx.doi.org/10.11609/JoTT.03682.6576-81

Copyright: (c) Karuthapandi 2014. Creative Commons Attribution 4.0 International License. JoTT allows unrestricted use of this article in any medium, reproduction and distribution by providing adequate credit to the authors and the source of publication.

Funding: Funding by Zoological Survey of India to M. Karuthapandi, SRF.

Competing Interest: The authors declare no competing interests.
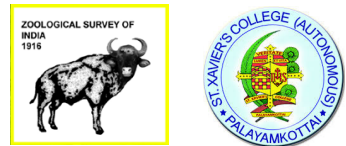

Acknowledgements: The authors are thankful to Dr. K. Venkataraman, Director, Zoological Survey of India, Kolkata, and the Officer-in-Charge, Freshwater Biology Regional Centre, Hyderabad for facilitates and constant encouragements. We also thank Dr. Y. Ranga Reddy, Emeritus Professor, ANU University, Nagarjunga Nagar, Guntur for his support in supplying literature on Ostracoda. 
the status of the uncertain species is marked with hash sign (\#) in the checklist. It is found that 50 species reported from India have been synonymised (Martens \& Savatenalinton 2011; Karanovic 2012) and their current valid nomenclature is also given in Table 3.

Literature reveals that there were several new species described in the past few decades from the Indian subcontinent by various workers, but the descriptions of most of the species seem to be insufficient to give the status of new species. Although most Indian taxonomists emphasized carapace morphology in ostracod identification, recent studies highlight that, in fact, soft parts morphology was crucial for their proper systematic placement. It was also observed that most of the species reported from India lack voucher and type locality information, which additionally contributes to the instability of the current Ostracoda nomenclature and validity of the species. Hence, the present checklist uses the type locality information provided in Karanovic (2012).

Freshwater faunal studies of Indian Ostracoda are limited and scattered, when compared to the global studies. In fact, the studies in this country are confined to a few states like Tamil Nadu, Kerala, Maharashtra, Madhya Pradesh, Chhattisgarh, Rajasthan, and a few parts of Delhi. India is one of the biodiversity rich countries in the world. The oriental region is the hotspot of ostracoda diversity with 200 species, of which $83 \%$

Table 1. Status of the freshwater Ostracoda of the world and India

\begin{tabular}{|c|l|c|c|c|}
\hline & & World & Oriental region & India \\
\hline 1 & Superfamilies & 4 & 1 & 2 \\
\hline 2 & Families & 15 & 6 & 5 \\
\hline 3 & Subfamilies & 34 & 16 & 15 \\
\hline 4 & Genera & 209 & 46 & 39 \\
\hline 5 & Species & 2103 & 200 & 152 \\
\hline
\end{tabular}

Table 2. Family wise Ostracoda species account from India

\begin{tabular}{|c|l|c|c|c|}
\hline & Families & World & Oriental region & India \\
\hline 1 & Cyprididae & 998 & 154 & 119 \\
\hline 2 & Candonidae & 545 & 17 & 12 \\
\hline 3 & Ilyocyprididae & 33 & 8 & 6 \\
\hline 4 & Notodromadidae & 36 & 15 & 14 \\
\hline 5 & Darwinulidae & 29 & 4 & 1 \\
\hline 6 & Limnocytheridae & 144 & 1 & - \\
\hline 7 & Laxoconchidae & 4 & 1 & - \\
\hline
\end{tabular}

(World and Oriental region data source from Martens et al. 2008) are endemic (Martens et al. 2008) and nearly $58 \%$ of the species are endemic to India within the oriental region. This study emphasizes the need for further detailed investigation to confirm the validity and status of the majority of ostracod species reported from India.

\section{Systematic list of Indian Freshwater Ostracoda}

Class: Ostracoda

Subclass: Prodocopa

Order: Podocopida

Suborder: Cypridocopina

Super family: Cypridoidea

\section{Family: Cyprididae}

Subfamily: Cyprettinae

1. Cypretta alagarkoilensis Victor \& Michael, 1975

2. Cypretta fontinalis Hartman, 1964

3. Cypretta foveata Hartmann, 1964

4. Cypretta gargi Deb, 1983

5. Cypretta globosa (Brady, 1886)

6. Cypretta globula (Sars, 1889)

7. Cypretta patialaensis Battish, 1982

8. Cypretta raciborskii (Grochmalicki, 1915)

9. Cypretta ramai Deb, 1984

10. Cypretta seurati Gauthier, 1929

11. Cypretta turgida Sars, 1896

12. Pseudocypretta maculata Klie, 1932

Subfamily: Cypricercinae

13. Astenocypris papyracea (Sars, 1903)

14. Bradleycypris vittata (Sars, 1903)

15. Bradleystrandesia dani (George \& Martens, 1993)

16. Bradleystrandesia mollis (Furtos, 1936)

17. Bradleystrandesia parva (Hartmann, 1964)

18. Bradleystrandesia reticulata (Zaddach, 1844)

19. Bradleystrandesia trichurensis (Victor et al., 1980)

20. Bradleystrandesia weberi (Moniez, 1892)

21. Bradleytriebella tuberculata (Hartmann, 1964)

22. Cypricercus indrani Deb, 1983 \#

23. Cypricercus munshii Deb and Nasar, 1977 \#

24. Cypricercus setosus Farkas, 1957

25. Cypricercus vietsi Farkas, 1957

26. Strandesia antetuberculata Hartmann \& Petersen, 1985

27. Strandesia bicornuta Hartmann, 1964

28. Strandesia flavescens Klie, 1932

29. Strandesia calapanensis Tressler, 1937

30. Strandesia gopinathani George \& Martens, 1993

31. Strandesia hartmanni Victor et al., 1980

32. Strandesia labiata Hartmann, 1964

33. Strandesia madhuriae Harshey \& Srinivasan 1984

34. Strandesia odiosa (Moniez, 1892) 
35. Strandesia perakensis Victor \& Fernando, 1981

36. Strandesia bornemiszai Klie, 1935

37. Strandesia purpurascens (Brady, 1886)

38. Strandesia quasirotunda Hartmann \& Petersen, 1985

39. Strandesia vinciguerrae (Masi, 1905)

40. Strandesia saetosa Hartmann, 1964

41. Strandesia victori Harshey \& Srinivasan, 1984

42. Tanycypris pellucida pellucida (Klie, 1932)

Subfamily: Cypridinae

43. Cypris debi Deb, 1983 \#

44. Cypris decaryi Gauthier, 1933

45. Cypris dravidensis Victor \& Michael, 1975 \#

46. Cypris elongata Deb, 1983 \#

47. Cypris globosa Deb, 1983 \#

48. Cypris kumari (Arora, 1931) \#

49. Cypris protubera Victor \& Fernando, 1978 \#

50. Cypris subglobosa Sowerby, 1840

51. Pseudocypris patialaensis Battish, 1977

Subfamily: Cypridopsinae

52. Cypridopsis maduraiensis Victor \& Michael, 1975

53. Klieopsis horai (Klie, 1927)

54. Plesiocypridopsis angularis (Victor \& Michael, 1975)

55. Plesiocypridopsis dispar (Hartmann, 1964)

56. Plesiocypridopsis newtoni (Brady \& Robertson, 1870)

57. Potamocypris dubia (Masi, 1905)

58. Potamocypris narayanani George \& Martens, 2002

59. Sarscypridopsis ochracea (Sars, 1924)

Subfamily Cyprinotinae

60. Cyprinotus cingalensis Brady, 1886

61. Hemicypris anomala (Klie, 1938)

62. Hemicypris arorai (Battish, 1981)

63. Hemicypris bairdi Martens \& Wouters 1985

64. Hemicypris bhatiai Battish, 1981

65. Hemicypris dentatomarginata (Baird, 1859)

66. Hemicypris derweshensis Battish, 1981

67. Hemicypris dissonus Victor \& Fernando, 1976

68. Hemicypris falcatus Victor \& Fernando 1976

69. Hemicypris gillensis (Battish, 1981)

70. Hemicypris malerkotlaensis Battish, 1981

71. Hemicypris megalops Sars, 1903

72. Hemicypris ovata Sars, 1903

73. Hemicypris pailensis Battish, 1981

74. Hemicypris pyxidata (Moniez, 1892)

75. Heterocypris anitae Battish, 1981

76. Heterocypris aurea Sars, 1895

77. Heterocypris chandrai (Arora, 1931)

78. Heterocypris fluviatilis (Furtos, 1933)

79. Heterocypris giesbrechti (G.W. Müller, 1898)
80. Heterocypris incongruens (Ramdohr, 1808)

81. Heterocypris indica (Battish, 1981)

82. Heterocypris makua (Tressler, 1937)

83. Heterocypris malini Deb, 1983

84. Heterocypris nuda (Victor \& Michael, 1975)

85. Heterocypris salina (Brady, 1868)

Subfamily: Dolerocypridinae

86. Dolerocypris sinensis Sars, 1903

Subfamily: Eucypridinae

87. Eucypris biharensis Deb, 1984

88. Eucypris bispinosa (Victor \& Michael, 1975)

89. Eucypris compressa Deb, 1983

90. Eucypris ellipticalis Deb, 1983

91. Eucypris gomti Deb, 1983

92. Eucypris himani Deb, 1983

93. Eucypris indica Deb, 1983

94. Eucypris inaequalis Deb, 1983

95. Eucypris munia Deb, 1983

96. Eucypris sonia Deb, 1983

Subfamily: Herpetocypridinae

97. Chrissia achandii (George \& Martens, 1993)

98. Chrissia biswasi (Deb, 1972)

99. Chrissia canaliculata (Hartmann, 1964)

100. Chrissia dyalpurensis Battish, 1998

101. Chrissia fasciculata (Daday, 1910)

102. Chrissia formosa (Klie, 1938)

103. Chrissia fuelleborni (Daday, 1910)

104. Chrissia goddeerisi (George et al., 1993)

105. Chrissia halyi (Ferguson, 1969)

106. Chrissia hislopi (Ferguson, 1969)

107. Chrissia humilis (Klie, 1932)

108. Chrissia humilis indica Hartmann, 1964

109. Chrissia khopoliensis (Deb, 1983)

110. Chrissia krishnakantai (Deb, 1972)

111. Chrissia spinosa (Tressler, 1937)

112. Herpetocypris ludhianensis Battish, 1982

113. Humphcypris sewelli (Klie, 1927)

114. Stenocypris derupta Vavra, 1906

115. Stenocypris distincta Victor \& Fernando, 1978

116. Stenocypris jabalpurensis Harshey \& Patil, 1988

117. Stenocypris major (Baird, 1859)

118. Stenocypris sohni Deb, 1983

Subfamily: Megalocypridinae

119. Sclerocypris rajasthaniensis Deb, 1973

Family: Candonidae

Subfamily: Candoninae

120. Candonopsis (Candonopsis) putealis Klie, 1932

121. Candonopsis (Candonopsis) urmilae Gupta, 1988

122. Indocandona krishnakanti Gupta, 1984

123. Indocandona nagarjuna Karanovic \& Ranga

Reedy, 2008 
Subfamily: Cyclocypridinae

124. Cypria brevisetigera Cole, 1965

125. Cypria devai (Arora, 1931)

126. Cypria javana javana G.W. Müller, 1906

127. Cypria minutua (Victor \& Michael, 1975)

128. Cypria sharmai Battish, 1985

129. Physocypria armata (G.W. Muller, 1897)

130. Physocypria crenulata (Sars, 1903)

131. Physocypria furfuracea (Brady, 1886)

Family: Ilyocyprididae

Subfamily: Ilyocypridinae

132. Ilyocypris australiensis Sars, 1889

133. Ilyocypris bradyi Sars, 1890

134. Ilyocypris dentifera Sars, 1903

135. Ilyocypris gibba (Ramdohr, 1808)

136. Ilyocypris mckenziei Bhatia \& Mannikeri, 1975

137. Ilyocypris nagamalaiensis Victor \& Michael, 1975

Family: Notodromadidae

Subfamily: Indiacypridinae

138. Indiacypris chalakkudensis George \& Martens,

2004.

139. Indiacypris dispar Hartman, 1964

Subfamily: Notodromadinae

140. Centrocypris bhagirathiae Battish, 1981

141. Centrocypris horrida (Vavra, 1895)

142. Centrocypris indica Gupta, 1991

143. Centrocypris madani Battish, 1981

144. Centrocypris matthaii (Arora, 1931)

145. Gurayacypris kangraensis Battish, 1987

146. Newnhamia dumonti George \& Martens, 2004

147. Newnhamia fenestrata King, 1855

148. Notodromas serrata Deb, 1984

Subfamily: Oncocypridinae

149. Oncocypris bhatiai Battish, 1982

150. Oncocypris voeltzkowi G.W. Muller, 1898

151. Sataracypris gibbosa (Baird, 1837) \#

Super family: Darwinuloidea

\section{Family: Darwinulidae}

152. Darwinula stevensoni (Brady \& Robertson, 1885)

\# Uncertain species

\section{References}

Arora, G.L. (1931). Fauna of Lahore 2 Entomostraca (Water fleas) of Lahore. Bulletin of the Department of Zoology University of the Punjab 1: 62-100.

Baird, W. (1859). Description of some new recent Entomostraca from Nagpur collected by Rev. S. Hislope. Proceedings of the Zoological Society of London 1859: 213-234.

Battish, S.K. (1978). A new genus and new species of Cypridid Ostracod from India, with notes on its biology. Zoological Journal of the Linnean Society 64: 283-292; http://dx.doi.org/10.1111/j.1096-3642.1978. tb01074.x

Battish, S.K. (1981). Freshwater Ostracods of the subfamily Cyprinotinae from Punjab, India with description of eight new species. Journal of Natural History Society 15: 645-669; http:// dx.doi.org/10.1080/00222938100770461

Battish, S.K. (1998). A new species of Cypridid Ostracod from India. Proceedings of the Zoological Society of Calcutta 52(1): 33-37.

Brehm, V. (1950). Contributions to the freshwater fauna of India. Records of the Indian Museum 48(2): 9-28.

Deb, M. (1972). Two new species of Stenocypris Sars, 1889. Journal of the Zoological Society of India 1: 91-95.

Deb, M. (1973a). First record of the genus Sclerocypris, Sars 1924 (Crustacea: Ostracods) from India with description of two new species. Journal of the Zoological Society of India 25: 47-52.

Deb, M. (1973b). Fauna of Rajasthan (India): Crustacea-Ostracoda. Records of the Zoological Survey of India 67: 233-259.

Deb, M. (1973c). Notes on a collection of Ostracoda from Delhi. Records of the Zoological Survey of India 67: 309-313.

Deb, M. (1983). Brief descriptions of new species on Ostracoda: Crustacea from Maharashtra state (India). Records of the Zoological Survey of India 81: 135-166.

Dudgeon, D., A.H. Arthington, M.O. Gessner, Z. Kawabata, D.J Knowler, C. Leveque, R.J. Naiman, A. Prieur-Richared, D. Soto, M.L.J. Stiassny, \& C.A. Sullivan (2006). Freshwater Biodiversity: Importance, threats, status and conservation challenges. Biological Reviews 81: 163-182.

George, S., K. Martens \& C.K.G. Nayar (1993). Two new species of freshwater Ostracoda of the genus Parastenocypris Hartmann, 1964 from Kerala, India. Hydrobiologia 254: 183-193; http://dx.doi. org/10.1007/BF00014112

George, S. \& K. Martens (2002). On a new species of Potamocypris (Crustacea, Ostracoda) from Chalakkudy River, Kerala (India), with a checklist of the Potamocypris species of the world. Zootaxa 66: 1-15.

George, S. \& K. Martens (2003). On a new species of the genus Newnhamia King, 1855 (Crustacea, Ostracoda) raised from Chalakkudy River sand, (Kerala, India), with notes on the taxonomy and distribution of the Notodromadidae. Hydrobiologia 497: 25-37; http://dx.doi.org/10.1023/a:1025453302991

George, S \& K. Martens (2004). On the taxonomic position of Indiacypridinae (Crustacea, Ostracoda) with description of a new species of Indiacypris Hartmann, 1964 from Chalakkudy River (Kerala, India). Journal of Natural History 38: 537-548; http:// dx.doi.org/10.1080/0022293021000013870

Gupta, L.P. (1984). Indocandona krishnakanti gen. et. sp. nov. (Crustacea: Ostracoda: Candonidae) from subterranean water of Bihar, India. Records of the Zoological Survey of India 81: 291-298.

Gupta, L.P. (1988). Candonopis urmilae, a new species of subterranean Crustacea (Ostracoda: Candonidae) from India. Records of the Zoological Survey of India 85: 419-427.

Gupta, L.P. (1991). Studies on Crustacea (Ostracoda) of Bihar, India. II. Centrocypris indicus sp. nov. Records of the Zoological Survey of India 89: 147-153.

Harshey, D.K. (1996). On some new records of fresh water Ostracods (Crustacea: Entomostraca) from India. Records of the Zoological Survey of India 95(3-4): 215-227.

Harshey, D.K. (2008). Crustacea (Ostracoda). Faunal Diversity of Jabalpur District, Madhya Pradesh. Edited by the Director, Zoological Survey of India, Kolkata. 63-75.

Harshey, D.K. \& J. Thilak (2011). Freshwater Ostracods (Arthropoda: Creustacea). Zoological Survey of India, Fauna of Madhya Pradesh (including Chhattisgarh), State Fauna Series 15(3): 31-44.

Harshey, D.K. \& R. Victor (1983). On Parastenocypris biswasi (Deb), 1972-A freshwater Ostracoda (Crustacea: Ostracoda) from India. Mitteilungen aus dem Hamburgischen Zoologischen Museum und Institut 80: 177-182.

Harshey, D.K. \& A.K. Srinivasan (1984). A new species of Strandesia Vavra, 1895 (Crustacea: Ostracoda) from India. Biovigyanam 13(2): 104-106. 
Table 3. List of Indian freshwater Ostracoda current valid names and synonyms

\begin{tabular}{|c|c|c|}
\hline & Current valid Name & Synonyms \\
\hline 1 & $\begin{array}{l}\text { Bradleycypris vittata (Sars, } \\
\text { 1903) }\end{array}$ & Cypris vittata Sars, 1903 \\
\hline 2 & $\begin{array}{l}\text { Bradleystrandesia mollis (Furtos, } \\
\text { 1936) }\end{array}$ & Cypricercus mollis Furtos, 1936 \\
\hline 3 & $\begin{array}{l}\text { Bradleystrandesia reticulata } \\
\text { (Zaddach, 1844) }\end{array}$ & Cypris reticulata Zaddach, 1844 \\
\hline 4 & $\begin{array}{l}\text { Bradleystrandesia trichurensis } \\
\text { (Victor et al., 1980) }\end{array}$ & $\begin{array}{l}\text { Strandesia trichurensis Victor et } \\
\text { al., } 1980\end{array}$ \\
\hline 5 & $\begin{array}{l}\text { Bradleystrandesia weberi } \\
\text { (Moniez, 1892) }\end{array}$ & Strandesia weberi Moniez, 1982 \\
\hline 6 & $\begin{array}{l}\text { Bradleytriebella tuberculata } \\
\text { (Hartmann, 1964) }\end{array}$ & $\begin{array}{l}\text { Strandesia tuberculata } \\
\text { Hartmann, } 1964\end{array}$ \\
\hline 7 & $\begin{array}{l}\text { Candonopsis (Candonopsis) } \\
\text { putealis Klie, } 1932\end{array}$ & Candonopsis putealis Klie, 1932 \\
\hline 8 & $\begin{array}{l}\text { Centrocypris matthaii (Arora, } \\
\text { 1931) }\end{array}$ & Eucypris matthaii Arora, 1931 \\
\hline 9 & $\begin{array}{l}\text { Chrissia achandii (George \& } \\
\text { Martens, 1993) }\end{array}$ & $\begin{array}{l}\text { Parastenocypris achandii } \\
\text { George \& Martens, } 1993\end{array}$ \\
\hline 10 & Chrissia biswasi (Deb, 1972) & $\begin{array}{l}\text { Parastenocypris biswasi (Deb, } \\
\text { 1972) } \\
\text { Stenocypris biswasi Deb, } 1972\end{array}$ \\
\hline 11 & $\begin{array}{l}\text { Chrissia canaliculata (Hartmann, } \\
\text { 1964) }\end{array}$ & $\begin{array}{l}\text { Parastenocypris canaliculata } \\
\text { Hartmann, } 1964\end{array}$ \\
\hline 12 & $\begin{array}{l}\text { Chrissia fasciculata (Daday, } \\
\text { 1910) }\end{array}$ & $\begin{array}{l}\text { Stenocypris fasciculata Daday, } \\
1910\end{array}$ \\
\hline 13 & Chrissia fulleborni (Daday, 1910) & $\begin{array}{l}\text { Stenocypris fulleborni Daday, } \\
1910\end{array}$ \\
\hline 14 & $\begin{array}{l}\text { Chrissia goddeerisi (George, } \\
\text { Martens \& Nayar, 1993) }\end{array}$ & $\begin{array}{l}\text { Parastenocypris goddeerisi } \\
\text { George, Martens \& Nayar, } 1993\end{array}$ \\
\hline 15 & Chrissia hislopi (Ferguson, 1969) & $\begin{array}{l}\text { Stenocypris hislopi Ferguson, } \\
1969\end{array}$ \\
\hline 16 & $\begin{array}{l}\text { Chrissia khopoliensis (Deb, } \\
\text { 1983) }\end{array}$ & $\begin{array}{l}\text { Stenocypris khopoliensis Deb, } \\
1983\end{array}$ \\
\hline 17 & Chrissia spinosa (Tressler, 1937) & $\begin{array}{l}\text { Stenocypris spinosa Tressler, } \\
1937\end{array}$ \\
\hline 18 & Cypris decaryi Gauthier, 1933 & Cypris ravenala Brehm, 1934 \\
\hline 19 & Cypris kumari (Arora, 1931) & Eurcypris kumari Arora, 1931 \\
\hline 20 & $\begin{array}{l}\text { Eucypris bispinosa (Victor \& } \\
\text { Michael, 1975) }\end{array}$ & $\begin{array}{l}\text { Cyprinotus bispinosa Victor \& } \\
\text { Michael, } 1975\end{array}$ \\
\hline 21 & Hemicypris ovata Sars 1903 & Cyprinotus ovatus (Sars, 1903) \\
\hline 22 & Heterocypris aurea (Sar, 1895) & Cyprinotus aureus Sars, 1895 \\
\hline 23 & $\begin{array}{l}\text { Heterocypris chandrai (Arora, } \\
\text { 1931) }\end{array}$ & Cyprinotus chandrai Arora, 1931 \\
\hline 24 & $\begin{array}{l}\text { Heterocypris fluviatilis (Furtos, } \\
\text { 1933) }\end{array}$ & $\begin{array}{l}\text { Cyprinotus fluviatilis Furtos, } \\
1933\end{array}$ \\
\hline 25 & $\begin{array}{l}\text { Heterocypris giesbrechtii (G. W. } \\
\text { Muller, 1898) }\end{array}$ & $\begin{array}{l}\text { Cyprinotus giesbrechtii G. W } \\
\text { Muller, } 1898 \\
\text { Cyprinotus gunning (Methuen, } \\
\text { 1910) }\end{array}$ \\
\hline
\end{tabular}

\begin{tabular}{|c|c|c|}
\hline 26 & $\begin{array}{l}\text { Heterocypris incongruens } \\
\text { (Ramdohr, 1808) }\end{array}$ & $\begin{array}{l}\text { Cyprisnotus incongruens } \\
\text { Ramdohr, } 1808\end{array}$ \\
\hline 27 & $\begin{array}{l}\text { Heterocypris indica (Battish, } \\
\text { 1981) }\end{array}$ & Cyprinotus indica Battish, 1981 \\
\hline 28 & $\begin{array}{l}\text { Heterocypris makua (Tressler, } \\
\text { 1937) }\end{array}$ & Cyprinotus makua Tressler, 1937 \\
\hline 29 & Heterocypris malini (Deb, 1983) & Cyprinotus malini Deb, 1983 \\
\hline 30 & $\begin{array}{l}\text { Heterocypris megalops Sars, } \\
1903\end{array}$ & $\begin{array}{l}\text { Cyprinotus megalops (Sars, } \\
\text { 1903) }\end{array}$ \\
\hline 31 & $\begin{array}{l}\text { Heterocypris nudus (Victor \& } \\
\text { Michael, 1975) }\end{array}$ & $\begin{array}{l}\text { Cyprinotus nudus Victor \& } \\
\text { Michael, } 1975\end{array}$ \\
\hline 32 & $\begin{array}{l}\text { Heterocypris salina (Brady, } \\
\text { 1868) }\end{array}$ & Cyprinotus salina Brady, 1868 \\
\hline 33 & $\begin{array}{l}\text { Himicypris gillensis (Battish, } \\
\text { 1981) }\end{array}$ & $\begin{array}{l}\text { Heterocypris gillensis (Battish, } \\
\text { 1981) }\end{array}$ \\
\hline 34 & Humphcypris sewelli (Klie, 1927) & Stenocypris sewelli Klie, 1927 \\
\hline 35 & $\begin{array}{l}\text { Indiacypris dispar Hartmann, } \\
1964\end{array}$ & $\begin{array}{l}\text { llyocypris dispar Hartmann, } \\
1964\end{array}$ \\
\hline 36 & Klieopsis horai (Klie, 1927) & Cypridopsis horai Klie, 1927 \\
\hline 37 & $\begin{array}{l}\text { Oncocypris voeltzkowi G. W. } \\
\text { Muller, } 1898\end{array}$ & $\begin{array}{l}\text { Oncocypris pustulosa Gurney, } \\
1916\end{array}$ \\
\hline 38 & $\begin{array}{l}\text { Plesiocypridopsis angularis } \\
\text { (Victor \& Michael, 1975) }\end{array}$ & $\begin{array}{l}\text { Cypridopsis angularis Victor \& } \\
\text { Michael, } 1975\end{array}$ \\
\hline 39 & $\begin{array}{l}\text { Plesiocypridopsis dispar } \\
\text { (Hartmann, 1964) }\end{array}$ & $\begin{array}{l}\text { Cypridopsis dispar Hartmann, } \\
1964\end{array}$ \\
\hline 40 & $\begin{array}{l}\text { Plesiocypridopsis newtoni (Brady } \\
\text { \& Robertson, 1870) }\end{array}$ & $\begin{array}{l}\text { Cypridopsis newtoni Brady \& } \\
\text { Robertson, } 1870\end{array}$ \\
\hline 41 & $\begin{array}{l}\text { Potamocypris dubia (Masi, } \\
\text { 1905) }\end{array}$ & Cypridopsis dubia Masi, 1905 \\
\hline 42 & $\begin{array}{l}\text { Pseudocypris dravidensis (Victor } \\
\text { \& Michael, 1979) }\end{array}$ & $\begin{array}{l}\text { Cypris dravidensis Victor \& } \\
\text { Michael, } 1979\end{array}$ \\
\hline 43 & $\begin{array}{l}\text { Sclerocypris rajasthaniensis } \\
\text { (Deb, 1973) }\end{array}$ & $\begin{array}{l}\text { Bharatcypris indica Deb, } 1983 \\
\text { Bharatcypris mackenziei Battish, } \\
1978 \\
\text { Bharatcypris dentata (Victor \& } \\
\text { Michael, 1975) }\end{array}$ \\
\hline 44 & $\begin{array}{l}\text { Strandesia pupurascens (Brady, } \\
\text { 1886) }\end{array}$ & $\begin{array}{l}\text { Strandesia meghnae Harshey \& } \\
\text { Shrivastava } 1987\end{array}$ \\
\hline 45 & $\begin{array}{l}\text { Strandesia victori Harshey \& } \\
\text { Srinivasan, } 1984\end{array}$ & $\begin{array}{l}\text { Strandesia neelimae Harshey \& } \\
\text { Shrivastava, } 1987\end{array}$ \\
\hline 46 & $\begin{array}{l}\text { Sarscypridopsis ochracea (Sars, } \\
\text { 1924) }\end{array}$ & Cypridopsis ochracea Sars, 1924 \\
\hline 47 & Stenocypris major (Baird, 1859) & $\begin{array}{l}\text { Stenocypris cylindrica major } \\
\text { Baird, } 1859\end{array}$ \\
\hline 48 & $\begin{array}{l}\text { Strandesia bornemiszai Klie, } \\
1935\end{array}$ & Strandesia prava Klie, 1935 \\
\hline 49 & $\begin{array}{l}\text { Strandesia calapanensis Tressler, } \\
1937\end{array}$ & $\begin{array}{l}\text { Strandesia elongata Hartmann, } \\
1964 \\
\text { Strandesia indica Hartmann, } \\
1964\end{array}$ \\
\hline 50 & $\begin{array}{l}\text { Strandesia vinciguerrae (Masi, } \\
\text { 1905) }\end{array}$ & $\begin{array}{l}\text { Strandesia rotunda (Tressler, } \\
\text { 1950) }\end{array}$ \\
\hline
\end{tabular}

Harshey, D.K \& A.K. Shrivastava (1983). Some new recodes of freshwater ostracods from India. Current Science 52(15): 741-742. Harshey, D.K., A.K. Shrivastava \& S.G. Patil (1987). Three new species of Strandesia Vavra, 1895 (Crustacea: Entomostraca) from India. Journal of Current Bioscience 45(2): 43--48.

Harshey, D.K \& S.G. Patil (1988). New species of Strandesia jabalpurensis from India. Journal of Biosciences 5(1): 6-10.

Hartmann, G. (1964). Asiatische Ostracoden, Systamatische und Zoogeographische untersuchungen. Internationale Revue der

Gesamten Hydrobiologie und Systematische Beihefte 3: 1-155.

Horne, D.J., A. Cohen \& K. Martens (2002). Taxonomy, morphology and biology of quaternary and living ostracoda, pp. 5-36. In: Holmes J.A. \& A.R. Chivas (eds.). The Ostracoda Applications in Quaternary Research. Geophysics Monograph-131.

Jayasree, T., K.S. Rao, K.A. Singh \& K. Sunil (1994). Observations on the Ostracoda fauna of central India with special reference to the western Narmada basin. Journal of Aquatic Biology and Fisheries 1(2): 41-50. 
Karanovic, I. (2012). Recent freshwater Ostracods of the World Crustacea, Ostracoda, Podocopida. Published by Springer, London. 1-608.

Karanovic, I. \& Y.R. Reddy (2008). The second representatives of the genus Indocandona Gupta (Crustacea, Ostracoda) from a well in southestern India. Crustaceana 72: 603-610

Klie, W. (1927). Zur ke nutnis der Mikrofauna von British Inidan. Records of the Indian Museum 1: 157-165.

Martens, K \& Savatenalinton (2011). Monograph: A subjective checklist of the Recent, free-living non-marine Ostracoda (Crustacea). Zootaxa 2855: 1-79.

Martens, K., I. Schon, C. Meisch \& D.J. Horne (2008). Global diversity of Ostracods (Ostracoda, Crustacea) in freshwater. Hydrobiologia 595: 185-193; http://dx.doi.org/10.1007/s10750-007-9245-4

Martin, J.W. \& G.E. Davis (2001). An updated classification of the recent Crustacea. Natural History Museum of Los Angeles, Science series 39: 1-124.

Patil, S.G. (2002). Freshwater Ostracoda, pp. 87-90. In: Fauna of Ujani. Zoological Survey of India, Wetland Ecosystem Series 3.

Patil, S.G \& S.S. Talmale (2005a). The freshwater Ostracoda, pp. 6375. In: Anon. (ed.). Fauna of Nathsagar Wetland. Zoological Survey of India, Wetland Ecosystem Series 7.
Patil, S.G. \& S.S. Talmale (2005b). A checklist of freshwater Ostracods (Ostracoda: Crustacea) of Maharashtra, India. Zoos' Print Journal 20(5): 1872-1873; http://dx.doi.org/10.11609/JoTT.ZPJ.1007.1872-

Patil, S.G. \& S.S. Talmale (2012). Freshwater Ostracods, pp. 309319. In: Director (ed.). Fauna of Maharashtra Part-2 Invertebrate. Zoological Survey of India, State Fauna series 20.

Venkataraman, K. (1999). Three species of freshwater Ostracoda (Crustacea) from Tamilnadu. Records of the Zoological Survey of India 97(3): 91-96.

Venkataraman, K. \& Krishnamoorthy (1998). Crustacea - Faunal Diversity in India. Published by ENVIS center, Zoological survey of India, Calcutta, 134-144.

Victor, R. \& C.H. Fernando (1979). The freshwater Ostracods (Crustacea: Ostracoda) of India. Records of the Zoological Survey of India 74(2): 147-242.

Victor, R. \& C.H. Fernando (1981). Freshwater Ostracods (Crustacea: Ostracoda) of the subfamily Dolerocypridinae Triebel, 1961 from Southeast Asia. Zoological Journal of the Linnean Society 72: 107116.

Victor, R. \& R.G. Michael (1975). Nine new species of freshwater Ostracods from Madurai area in southern India. Florida Museum of Natural History 9: 361-376; http://dx.doi. org/10.1080/00222937500770261 